\title{
Fundamental Investigations Regarding Barrier Properties of Grafted PVOH Layers
}

\author{
Markus Schmid, ${ }^{1}$ Andreas Benz, ${ }^{1}$ Camelia Stinga, ${ }^{2}$ \\ Daniel Samain, ${ }^{2}$ and Klaus Peter Zeyer ${ }^{3}$ \\ ${ }^{1}$ Materials Development Department, Fraunhofer Institute for Process Engineering and Packaging IVV, \\ Giggenhauser Straße 35, 85354 Freising, Germany \\ ${ }^{2}$ BT3 Technologies, Impasse Bellevue 8, 38240 Meylan, France \\ ${ }^{3}$ Department of Applied Sciences and Mechatronics, Munich University of Applied Sciences, \\ Lothstraße 34, 80335 Munich, Germany
}

Correspondence should be addressed to Markus Schmid, markus.schmid@ivv.fraunhofer.de

Received 25 May 2012; Accepted 5 September 2012

Academic Editor: Benny Dean Freeman

Copyright (C) 2012 Markus Schmid et al. This is an open access article distributed under the Creative Commons Attribution License, which permits unrestricted use, distribution, and reproduction in any medium, provided the original work is properly cited.

\begin{abstract}
The current work focuses on fundamental investigations regarding the barrier properties of grafted PVOH layers produced by the Transfer Method. The layers (or papers) used for the different experiments were produced and grafted during the course of this work. Papers with different types of PVOH (different Mowiol types) were produced by coating. Experiments using different parameters (temperature, reaction duration, and concentration) were performed using the Transfer Method. Contact angle measurements and $\mathrm{Cobb}_{60}$ measurements were carried out on the grafted and untreated PVOH layers. Furthermore, the water vapour transmission rate of the PVOH layers was determined. The results of this work showed that the method of chromatogeny or chromatogenic chemistry improves the water vapour barrier properties of grafted PVOH layers enormously.
\end{abstract}

\section{Introduction}

These fundamental investigations regarding the barrier properties of grafted PVOH (polyvinyl alcohol) and EVOH (ethylene vinyl alcohol copolymer) layers aim to facilitate the design and development of an innovative ecofriendly, low-substrate, flexible paper for packaging from renewable resources and so reduce the packaging industry's reliance on barrier films derived from petroleum.

The technical challenge of this study is to develop a flexible paper with a multilayered structure that has barrier properties (against water, grease, oxygen, water vapour, and aromas) comparable to those of untreated plastic films (medium barrier) or treated plastic films (high barrier).

This work focuses on the chemical method called chromatogenic chemistry. Accordingly, one experimental method was used in this work: the Transfer Method.

The Transfer Method is the method used to investigate the barrier properties of grafted PVOH layers. It will be shown that fatty acid chlorides are promising reagents for this kind of reaction. Focus will also be put on determining the kinetics of this grafting process and the effect of temperature and the concentration of fatty acid chlorides on the esterification of paper.

The aim of this work was therefore to investigate a material or paper coated with $\mathrm{PVOH}$ or EVOH which has a hydrophobic character at the end of the process. A tool to realise this aim was experimental design.

Whatever materials are used to manufacture a packaging, there is always a desire for a higher barrier at lower cost and a lower environmental impact. Paper and paperboards are produced from renewable resources. These substrates in combination with knowledge-led design of the barrier strategy represent very promising materials for future packaging concepts.

The current reliance on multimaterial (composite) packaging materials such as foil and polymeric laminates bound to paper carry a financial penalty which could be significantly 
reduced with inline processes that enable the production of a monomaterial barrier. Furthermore, the environmental impact can be drastically lowered by utilising renewable materials to generate the required properties. Such materials reduce the dependency on oil-based packaging materials and lead to repulpable, recyclable, and biodegradable products. With regard to the barrier properties of packaging materials, the critical substances which can penetrate into the packaging and degrade the quality of the food $A=\pi r^{2}$ water vapour and oxygen in the surrounding air.

Water vapour passes through packaging and binds reversibly to food via hydrogen bonds. Good barrier properties against oxygen can be obtained by multilayer structures from synthetic polymers including the copolymer $\mathrm{EVOH}$ or polyvinylidene chloride (PVDC). Alternatively, excellent barrier properties can be obtained by the deposition of fine layers of inorganic compounds like $\mathrm{SiO}_{x}$. However, these surface modifications require complex technical processes (vacuum deposition, plasma) and expensive materials. Moreover, the polymeric structures that are involved are not biodegradable and cannot be recycled easily [1-3].

Evidence from the literature suggests that it is possible to produce barrier materials from renewable resources on a short to medium time-scale, provided adequate research is carried out to develop the products and adapt them to the process requirements. Nevertheless, two significant challenges need to be overcome to enable successful implementation $[4,5]$.

(i) Most biopolymers are hydrophilic and lose all their barrier properties when exposed to water or high humidity.

(ii) The oxygen and water vapour barrier level of biopolymers is still an order lower than that of barrier plastic films.

One innovative surface treatment method (the Transfer Method) will be developed to overcome these drawbacks and facilitate the production of a high-barrier flexible paper. A solvent-free chemical grafting process [6], which enables the covalent grafting of natural fatty acids onto the hydroxyl groups in the surface layers, will be used to impart strong hydrophobic properties to the paper.

\section{Materials and Methods}

2.1. Chemicals. Palmitoyl chloride (C16), stearoyl chloride (C18), and petroleum ether (boiling range $100-140^{\circ} \mathrm{C}$ ) were purchased from Merck KGaA (Darmstadt, Germany) and TCI Deutschland GmbH (Eschborn, Germany). The different polyvinyl alcohol types (Poval 505, Mowiol 4-88, Mowiol 4-98 and Mowiol 28-99) and one ethylene vinyl alcohol copolymer (Exceval AQ4104) were kindly provided by Kuraray Europe GmbH (Frankfurt/Main, Germany).

2.2. Laboratory Equipment/Materials. Whatman filter paper No. 2 (Whatman GmbH, Dassel, Germany) was used as the cellulosic substrate and SCC (sized coated calendered) paper

$$
\begin{aligned}
& \mathrm{R}-\mathrm{OH}+\mathrm{R}^{\prime} \mathrm{COOX} \underset{k_{2}}{\stackrel{k_{1}}{\longleftarrow}} \mathrm{ROOCR}^{\prime}+\mathrm{HX} \\
& \mathrm{R}:\left[\mathrm{CH}_{3}-\mathrm{CH}_{2}\right]_{n} \\
& \mathrm{R} \text { : }\left[\mathrm{CH}_{3}-\mathrm{CH}_{2}\right]_{m} \\
& \mathrm{X}: \text { halogen }
\end{aligned}
$$

FIGURE 1: Reaction equation for chromatogenic chemistry.

was used as the coating substrate. An oven with adjustable air flow and temperature was used for the Transfer Method.

2.3. Transfer Method. The performance of the Transfer Method was investigated by Berlioz [7] on Whatman paper number 2. It was shown that the transport mechanism of diffusion of the reagent is very important for this method. Based on these tests, the Transfer Method was further developed. This included the grafting of materials onto substrates which is achieved by gas diffusion of the fatty acid chloride to the coated paper [1].

Generally, the reaction equation for chromatogenic chemistry is shown in Figure 1.

The overall reaction consists of a reactant side and a product side. The reactants are $\mathrm{R}-\mathrm{OH}$, a type of polyvinyl alcohol, and $\mathrm{R}^{\prime} \mathrm{COOX}$ which signifies a type of fatty acid chloride. There are two products. ROOCR' presents the general structure of the ester which is significant for the hydrophobicity and chromatogenic chemistry. Furthermore, HX stands for a hydrogen halide (e.g., hydrogen chloride) as used in this study. It is the only gas which develops during the grafting process.

The procedure of chromatogeny and therefore the process of the Transfer Method will now be described. The reagent is applied in liquid form, finely dispersed on the surface of a porous material (here: the paper) which is then subjected to a hot, inert gas flow which influences the reaction. The existence of a liquid-vapour equilibrium enables the reagent to diffuse, due to the gas flow, inside porous materials by a mechanism of adsorption-desorption. The gas flow moves the molecules in the vapour phase, causing a displacement of the chemical equilibrium.

This results in the diffusion of the reagent into the interior of the solid substrate. During this process of diffusion, grafting occurs. The reagent is adsorbed onto the surface of the substrate and reacts with available hydroxyl groups. The byproduct that is generated is removed by the gas flow, which avoids any possible degradation of the substrate $[1,8]$.

The Transfer Method was performed as follows. After preparation of the samples, the tests were started by coating the SCC papers. A blade of $100 \mu \mathrm{m}$ wet film thickness was used to deposit approximately $7.5 \mathrm{~mL}\left(15 \mathrm{~g} / \mathrm{m}^{2}\right.$ as application weight) of the $\mathrm{PVOH}$ type (concentration $=15 \%$ ) [9] on the paper. The only exception was Mowiol 28-99 which had a different viscosity than Mowiol 4-88 and Mowiol 4-98 and therefore the concentration for Mowiol 28-99 was set at 5\% [9]. The coated paper was then put in the oven at $150^{\circ} \mathrm{C}$ for 
6 minutes to dry all the PVOH. This is the first part of the Transfer Method.

Before beginning with the second step of the Transfer Method, the solution of fatty acid chloride and petroleum ether had to be prepared ( $2 \%$ fatty acid chloride, $98 \%$ petroleum ether).

The second step is the "main" process of the transfer experiment. The procedure for this is described below.

(1) Immerse the Whatman paper in the solution of fatty acid chloride (2\%) and petroleum ether (98\%).

(2) After a short drying period, the immersed paper is put on the coated PVOH layers or the coated EVOH layer.

(3) (a) Put the "two" papers in the oven to start the chemical grafting process

(b) The temperature of the oven and heating time were set beforehand with the help of Visual XSel experimental design.

(4) Step (3) finishes the process and the "finished paper" (after the grafting process) has a hydrophobic surface.

\section{Results and Discussion}

3.1. Results of the Transfer Method. The results of the Transfer Method are represented by graphs obtained from Visual XSel 11.0 analysis with PLS (partial least square) and Microsoft Excel. They show different relations between the parameters and also $3 \mathrm{D}$ graphs where 3 parameters are combined in one graph [10].

Figure 2 shows a 3D graph with a specific curve for FA C16 and Mowiol 28-99 as the PVOH type. The level of significance is $\alpha=5 \%$ and the coefficient of determination is $R^{2}=64 \%$ which means that $36 \%$ of undefined statistical spread remains. These values are very satisfactory for this kind of model because the measurements disperse or correlate often. This dispersion of the measurement values for the Transfer Method should allow adjustment of the parameters to get the desired results. One has to emphasize that Figure 2 only represents this type of FA and $\mathrm{PVOH}$ and not all FA and $\mathrm{PVOH}$ types. However, in order to see the general effect of the Transfer Method on the Cobb 60 , this figure is also representative for other FA and $\mathrm{PVOH}$ types.

The different parameters are represented in the following way:

(i) $x$-axis: temperature $\left({ }^{\circ} \mathrm{C}\right)$,

(ii) $y$-axis: $\operatorname{Cobb}_{60}\left(\mathrm{~g} / \mathrm{m}^{2}\right)$,

(iii) $z$-axis: time $(\min )$.

The blue coloured region signifies $\operatorname{Cobb}_{60}$ values ranging from 0 to approximately $6 \mathrm{~g} / \mathrm{m}^{2}$ (explanation in the legend). Generally, the $y$-axis values fall from 10 to $0 \mathrm{~g} / \mathrm{m}^{2}$ while the values on the $x$-axis increase from 100 up to $165^{\circ} \mathrm{C}$. It is shown that time has a big influence on the $\mathrm{Cobb}_{60}$ value. It is perfectly clear from this graph that the Transfer Method mostly yields $\mathrm{Cobb}_{60}$ values smaller than $6 \mathrm{~g} / \mathrm{m}^{2}$, whereas the untreated layers or papers have $\mathrm{Cobb}_{60}$ values around
$20 \mathrm{~g} / \mathrm{m}^{2}$. These differences between grafted and untreated papers can also be seen in Figure 5.

Another fact which can be seen is that the best values for $\mathrm{Cobb}_{60}$ were found for C16 as FA and Mowiol 28-99 as $\mathrm{PVOH}$. For example, at 6 minutes and $150^{\circ} \mathrm{C}$ a Cobb 60 value of nearly $0 \mathrm{~g} / \mathrm{m}^{2}$ was obtained.

This reading of the measurement values can be done for the whole figure and it is then possible to find specific $\operatorname{Cobb}_{60}$ values for each temperature in combination with time. With the help of this figure it is possible to say that the Transfer Method has an excellent effect on the surface of the paper because the water absorption of the grafted paper is virtually zero.

Figure 3 shows a 3D graph with C16 as FA and Mowiol 28-99 as PVOH. Essentially, the principles for the level of significance and the coefficient of determination are the same as for Figure 2.

The three axes are:

(i) $x$-axis: temperature $\left({ }^{\circ} \mathrm{C}\right)$,

(ii) $y$-axis: contact angle $\left({ }^{\circ}\right)$,

(iii) $z$-axis: time $(\mathrm{min})$.

Overall a rise in the curve from nearly $96^{\circ}$ up to approximately $109^{\circ}$ is visible. The contact angle rises in the temperature interval from $90^{\circ} \mathrm{C}$ to approximately $150^{\circ} \mathrm{C}$. Above $150^{\circ} \mathrm{C}$ a slight decline can be observed. In order to interpret the shape of the curve, this maximum is not significant enough to draw general conclusions. The decline can be reasoned by the evaporation and decomposition of the reagent.

It can be seen that the colours red and violet are dominant. Therefore, the majority of the contact angle values for this type of FA and PVOH are above $100^{\circ}$. Figures 5 and 6 show the comparison between untreated and grafted $\mathrm{PVOH}$ layers and the effect of the Transfer Method.

It can be stated that the Transfer Method has a large influence on the properties of the surface of the PVOH layers. The measurement values are all $>90^{\circ}$ which signifies that the surface of the paper has hydrophobic properties [11].

Figure 4 gives an overview of the contact angle results for the Transfer Method. The contact angle characterises the surface properties of a material. So in this case it serves as a way of saying whether a surface is hydrophilic or hydrophobic. The results in Figure 4 are the average values of the measurements. Generally, all values are higher than $90^{\circ}$ which signifies that the material has a hydrophobic character. The blue bars represent the different $\mathrm{PVOH}$ layers grafted with $\mathrm{C} 16$ and the red columns show the different $\mathrm{PVOH}$ layers grafted with $\mathrm{C} 18$. One aspect which can be observed is that Mowiol 28-99 has the highest contact angles values for both C16 and C18. By considering, for example, the course of the red columns from Poval 505 to Mowiol 28-99, a constant increase in the contact angles is observed. A reason for this observation can be found by looking at the molecular masses, the degrees of hydrolysis, and the viscosities of the different $\mathrm{PVOH}$ types. With increasing degree of hydrolysis, the density of esterification also increases. Furthermore, another important aspect is that the molecular mass increases with 


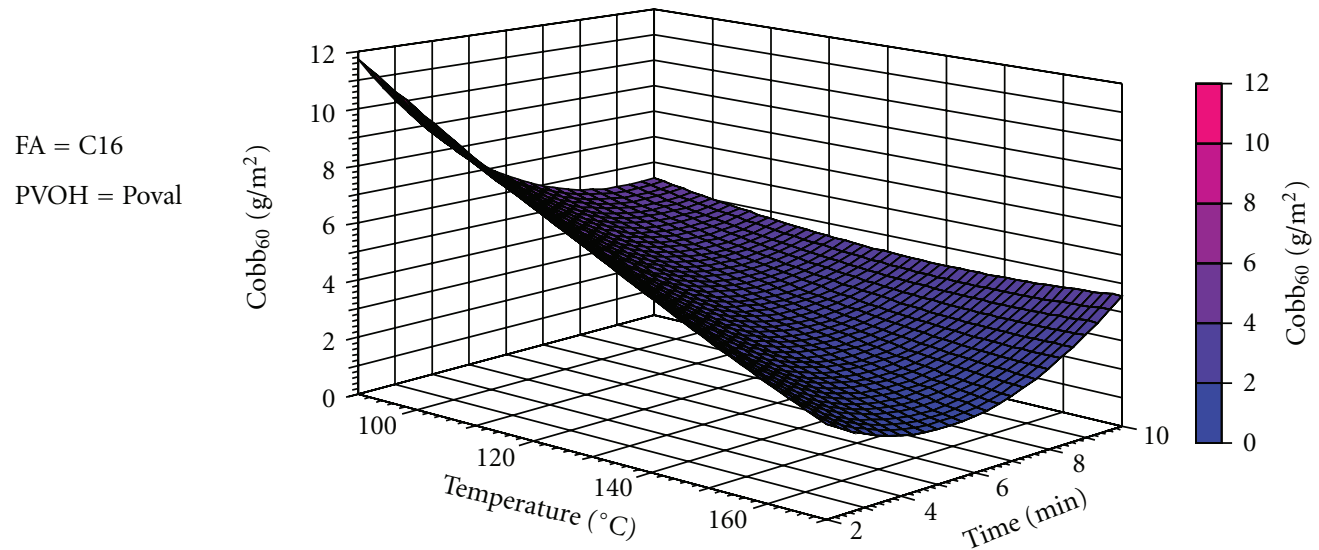

Figure 2: 3D graph showing $\operatorname{Cobb}_{60}$, temperature, and time.

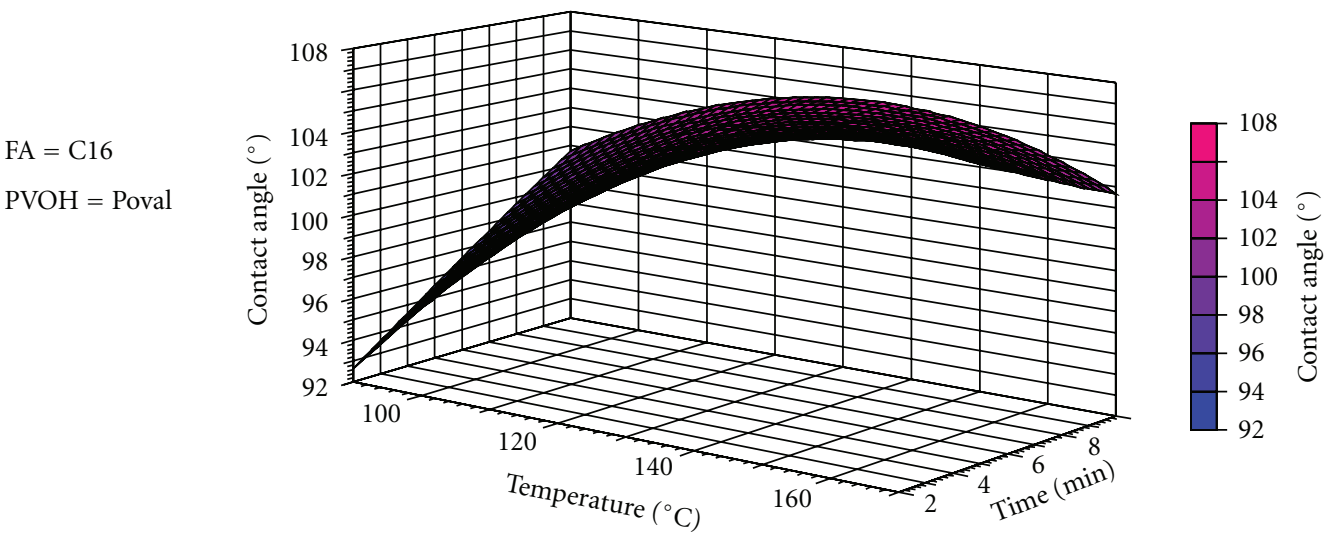

FIgURE 3: 3D graph showing contact angle, temperature, and time.

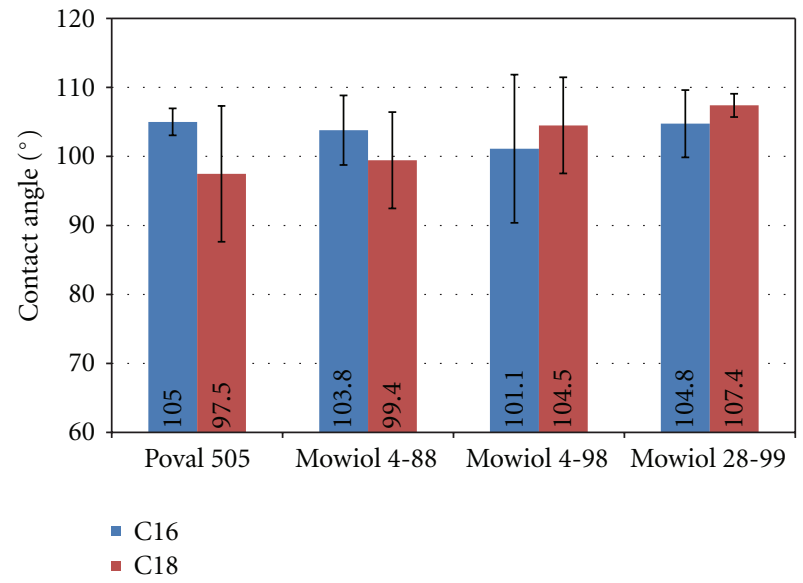

FIGURE 4: Comparison of the contact angle of grafted PVOH layers C16/C18.

increasing viscosity. Therefore, the higher the degree of hydrolysis and the higher the viscosity, the greater is the contact angle.

3.1.1. Results of the Cobb 60 Measurements. The Cobb 60 measurements were performed as outlined in the standard

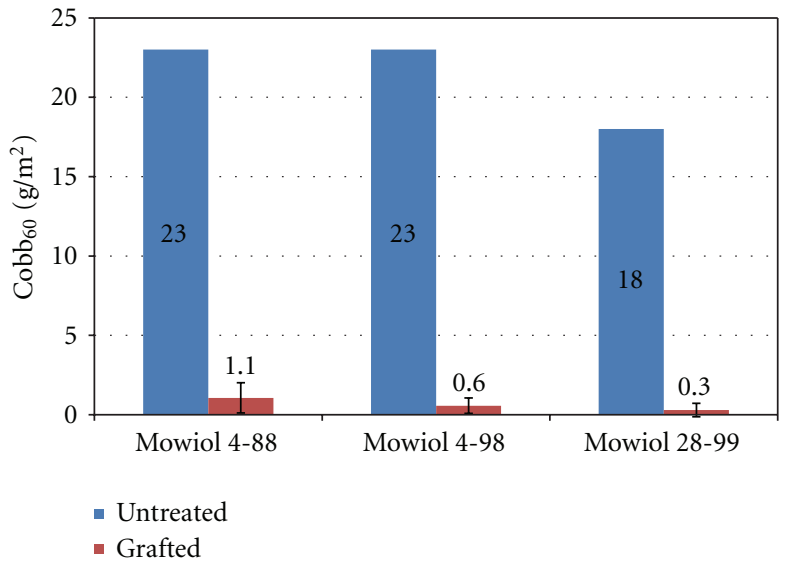

FIGURE 5: Comparison of $\mathrm{Cobb}_{60}$ values for untreated and grafted PVOH layers.

[12]. By comparing selected measurements for the untreated and grafted PVOH layers it can be observed that the $\mathrm{Cobb}_{60}$ $\left(\mathrm{g} / \mathrm{m}^{2}\right)$ is affected by the grafting process. The grafted papers have much lower Cobb $_{60}$ values than the untreated papers. By regarding one Mowiol type, for example Mowiol 28-99, 


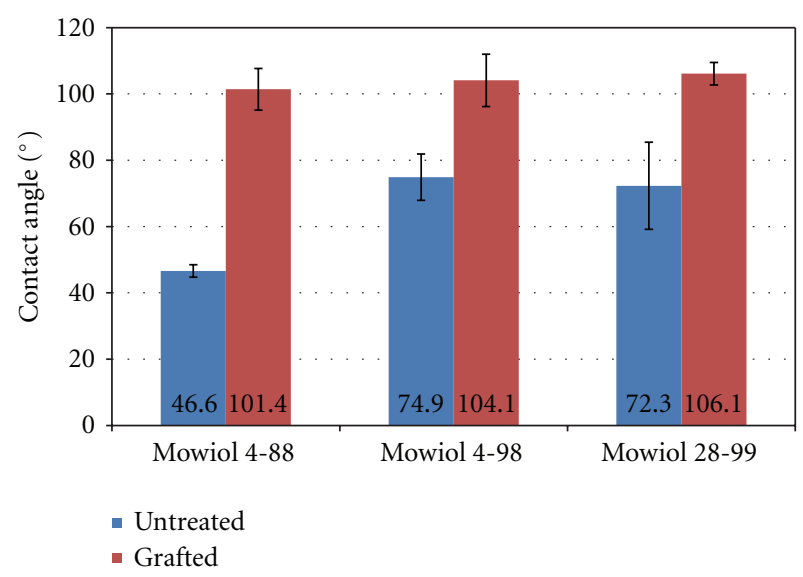

Figure 6: Comparison of contact angle values for untreated and grafted PVOH layers.

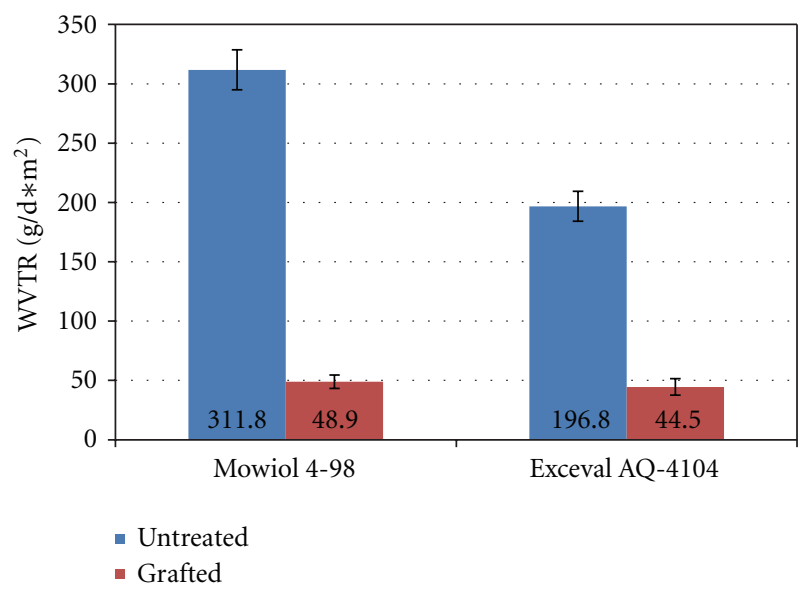

Figure 7: Comparison of the water vapour transmission rates for untreated and grafted $\mathrm{PVOH}$ and $\mathrm{EVOH}$ layers.

the measurement value for the untreated layer was $18 \mathrm{~g} / \mathrm{m}^{2}$. This value is much higher than the measurement for the grafted layer $\left(0.3 \mathrm{~g} / \mathrm{m}^{2}\right)$. The reason for this effect has already been explained and can also be seen in Figure 2. The results given in this section will facilitate the understanding of the results in the $3 \mathrm{D}$ graphs and provide an overview of the measured values.

3.1.2. Results of the Contact Angle Measurements. This section compares the contact angle measurements of the untreated and grafted $\mathrm{PVOH}$ layers, and so emphasises once again the effect of the Transfer Method.

Generally, all the contact angles for the grafted PVOH layers are above $90^{\circ}$ which means that the layers or papers have a hydrophobic surface. The effect of hydrophobicity is also seen in Figure 3. In contrast, the untreated PVOH layers have contact angles smaller than $90^{\circ}$. This means that the surface of the untreated papers is hydrophilic. In detail, the effect of the Transfer Method can be seen by considering Mowiol 28-99. The untreated layers had a contact angle of approximately $72.3^{\circ}$ which means that the surface of the material is hydrophilic. The surface of the grafted PVOH layers is hydrophobic because they have a contact angle about $106.1^{\circ}$.

In summary, the results showed that the Transfer Method affects the $\mathrm{PVOH}$ layers and furthermore the higher the degree of hydrolysis the higher is the contact angle. Therefore, the surface of the layer is more hydrophobic.

It can be stated that measurement of the contact angle is another method for validating the Transfer Method and showing its outstanding effect.

3.1.3. Results of the Water Vapour Measurements. The water vapour measurements were carried out at a relative humidity of $85 \%$ and a temperature of $23^{\circ} \mathrm{C}$ [13]. Figure 7 demonstrates the effects of the $\mathrm{PVOH}$ and $\mathrm{EVOH}$ layers produced by the grafting process on the water vapour transmission rate (WVTR) or permeation in $\mathrm{g} /\left(\mathrm{d} \cdot \mathrm{m}^{2}\right)$. Considering untreated Mowiol 4-98, the WVTR is about $312 \mathrm{~g} /\left(\mathrm{d} \cdot \mathrm{m}^{2}\right)$ and after the grafting process the permeation is about $49 \mathrm{~g} /\left(\mathrm{d} \cdot \mathrm{m}^{2}\right)$. So the difference between untreated and grafted Mowiol 4-98 is exactly $263 \mathrm{~g} /\left(\mathrm{d} \cdot \mathrm{m}^{2}\right)$ and, thus, the hydrophobic character of the grafted material is confirmed. The same results with other measurements can be obtained for the EVOH layer. So in total, the effect of the Transfer Method can be determined. Another associated and interesting aspect is the fact that the WVTR of Mowiol 4-98 decreases by a factor of approximately 6 and the WVTR of the EVOH decreases by a factor of only around 4. This can be explained by the fact that EVOH has a lower initial WVTR due to the non-polar ethylene segments in the polymer.

\section{Conclusions}

The objective of this work was to study the barrier properties of grafted PVOH layers. This included experiments to improve the water barrier function of $\mathrm{PVOH}$ layers which primarily have hydrophilic properties. In order to achieve hydrophobicity, the Transfer Method has been studied using different parameters (e.g., temperature and reaction duration). To validate the results of chromatogenic chemistry, various measurements have been made $\left(\mathrm{Cobb}_{60}\right.$ determination, contact angle measurement, and water vapour transmission rate) on selected samples.

The Transfer Method embodies chromatogenic chemistry because the surface of the paper coated with $\mathrm{PVOH}$ becomes hydrophobic.

The method, in conjunction with measurement of the contact angle, showed that on increasing the temperature the contact angle also increases. Namely, as the degree of hydrolysis increases so also does the contact angle.

$\mathrm{Cobb}_{60}$ measurements were also made. The $\mathrm{Cobb}_{60}$ values decreased when the temperature, and in some cases the processing time in the oven, was increased.

All the results show that as the degree of hydrolysis increases so also does the esterification density. Therefore, the effect of the Transfer Method is readily seen.

The Cobb $_{60}$, contact angle and water vapour transmission rate data were summarised. Comparisons between untreated and grafted PVOH layers were made. In this work 
the focus has been on measurement of the $\mathrm{Cobb}_{60}$ and the contact angle to quantify the hydrophobicity of PVOH layers after the grafting process.

In summary, the results of this work showed that the method of chromatogeny or chromatogenic chemistry improves the water vapour barrier properties of grafted PVOH layers enormously. Experimental design prior to the measurements was very helpful for having a well-structured experimental process. It also enabled a reduction in the number of experiments without losing the ability to interpret the results. In conclusion it can be stated that the grafted PVOH layers showed the desired effect of hydrophobicity and that the fundamental investigation of the barrier properties of these layers was successfully performed.

\section{Notations}

C16/18: 16/18 carbon atoms

EVOH: Ethylene vinyl alcohol

FA: $\quad$ Fatty acid chloride

PLS: Partial least square

PVOH: Polyvinyl alcohol

WVTR: Water vapour transmission rate, grams per day and square metre $\left[\mathrm{g} /\left(\mathrm{d} \cdot \mathrm{m}^{2}\right)\right]$.

\section{Authors' Contribution}

Markus Schmid and Andreas Benz contributed equally to this work.

\section{References}

[1] N. C. Stinga, Utilisation de la Chimie Chromatogénique Pour la Conception et la Réalisation de Matériaux Cellulosiques Barrières à L'eau, aux Graisses et aux Gaz, Université de Grenoble, 2008.

[2] S. I. Hong and J. M. Krochta, "Oxygen barrier properties of whey protein isolate coatings on polypropylene films," Journal of Food Science, vol. 68, no. 1, pp. 224-228, 2003.

[3] M. Schmid, K. Dallmann, E. Bugnicourt et al., "Properties of whey protein coated films and laminates as novel recyclable food packaging materials with excellent barrier properties," International Journal of Polymer Science, vol. 2012, Article ID 562381, 7 pages, 2012.

[4] H. J. Endres and A. Siebert-Raths, Technische Biopolymere Rahmenbedingungen, Marktsitutation, Herstellung, Aufbau Und Eigenschaften, Hanser, Munich, Germany, 2009.

[5] R. Chandra and R. Rustgi, "Biodegradable polymers," Progress in Polymer Science, vol. 23, no. 7, pp. 1273-1335, 1998.

[6] D. Samain, Procédé de Traitement d'un Matériau solide Pour le Rendre Hydrophobe, Matériau Obtenu et Applications, France, 1998.

[7] S. Berlioz, Étude de l'Estérification de la Cellulose par une Synthèse sans Solvant. Application aux Matériaux Nanocomposites, Université Joseph, Fourier, Grenoble, France, 2007.

[8] C. Stinga, D. Samain, and D. Guérin, Food Packaging-Eco Conception, in Chromatogenic Days, CTP, Grenoble, France, 2010.

[9] K. E. GmbH, Ed., Kuraray, Mowiol—Polyvinyl Alcohol, Frankfurt, Germany, 2003.
[10] C. Ronninger, Design of Experiments \& Statistics, Munich, Germany, 11th edition, 2010.

[11] S. Matthias, Kohlenstoff-Nanoröhrchen als Elektrodenmaterial für PEM-Brennstoffzellen [Ph.D. thesis], 2011.

[12] DIN53132, Bestimmung der Wasseraufnahme Nach Cobb, Beuth, Berlin, Germany, 1980.

[13] DIN53122, Bestimmung der WasserdampfdurchlässigkeitGravimetrisches Verfahren, Beuth, Berlin, Germany, 1974. 

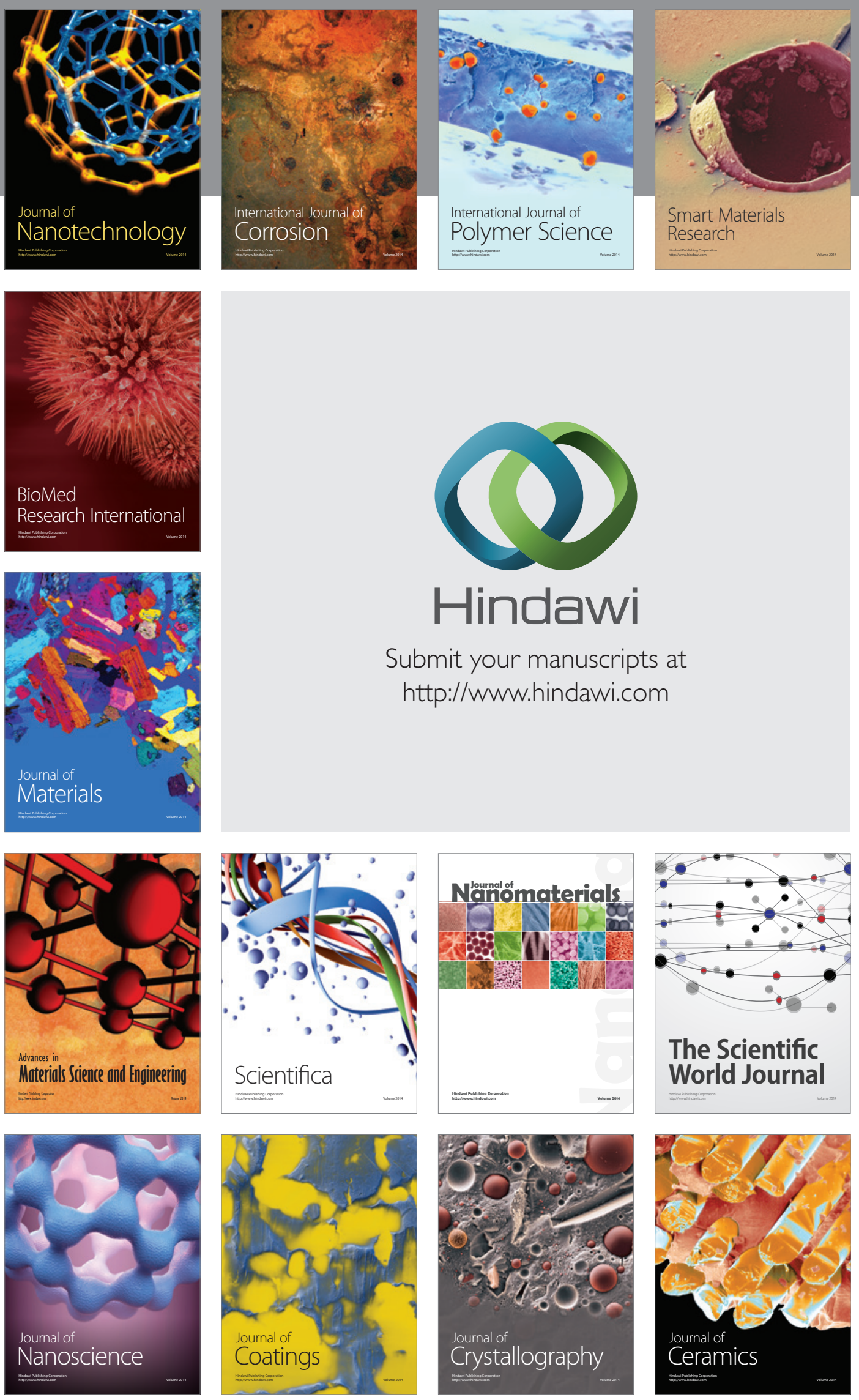

The Scientific World Journal

Submit your manuscripts at

http://www.hindawi.com

\section{World Journal}

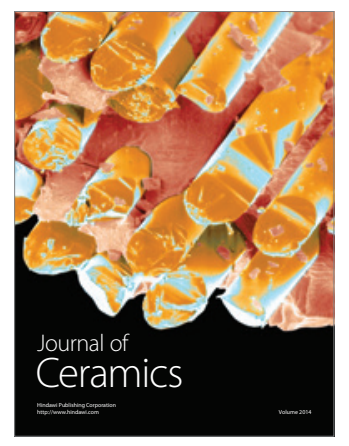

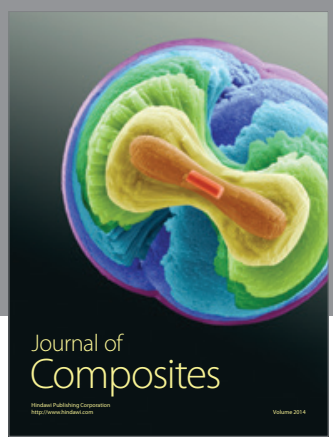
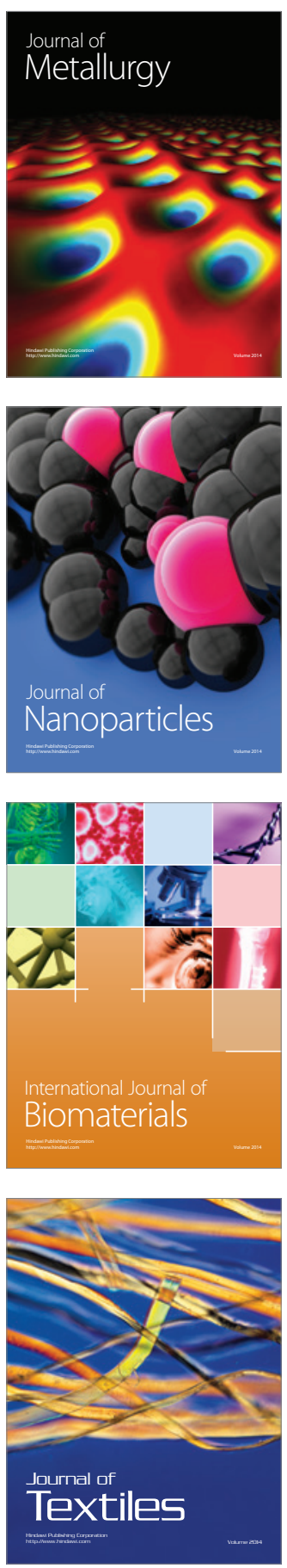